\title{
Effects of camber on wheeling efficiency in the experienced and inexperienced wheelchair user
}

\author{
Angeliki Perdios, MSc; ${ }^{1 *}$ Bonita J. Sawatzky, PhD; ${ }^{2}$ A. William Sheel, PhD ${ }^{1}$ \\ ${ }^{1}$ School of Human Kinetics and ${ }^{2}$ Department of Orthopaedics, The University of British Columbia, Vancouver, British \\ Columbia, Canada
}

\begin{abstract}
The objective of this study was to determine whether energy costs differed between $0^{\circ}, 3^{\circ}$, and $6^{\circ}$ of camber during steady state overground wheeling. Three subject groups were examined: experienced wheelchair users with disabilities (thoracic lesion level 6 and below), nondisabled individuals with manual wheeling experience, and nondisabled individuals with no manual wheeling experience. Heart rate, rating of perceived exertion, visual analog scale for comfort, and a user preference questionnaire were collected for all subjects. Expired gas analysis data were collected for the group with disabilities. No statistically significant differences emerged in respiratory measures for camber angle or group. A camber of 6 degrees was most preferred in terms of stability on a side slope, hand comfort on the pushrims, maneuverability, and overall preference. Rear-wheel camber angle did not affect the energy expenditure of manual wheelchair propulsion, as measured by cardiopulmonary means. The individual manual wheelchair user's perceived level of comfort should be the determining factor in rear-wheel camber selection.
\end{abstract}

Key words: adult, camber, efficiency, heart rate, oxygen consumption, paraplegia, physiology, rehabilitation, spinal cord injury, wheelchair, wheeling.

\section{INTRODUCTION}

Studies that have specifically investigated camber and energy cost have had differing results. Increased rear-wheel camber has some minor disadvantages, such as increased wheelbase and decreased wheelchair height, that may present problems when the user is negotiating obstacles [1-2]. However, the increased chair width improves turning stability; reduces downward turning tendency on side slopes; protects the hands since the base of the wheel is the widest part of the chair and will contact any obstacles first; and positions the wheelchair pushrims more ergonomically for propulsion, reducing the static effort of the shoulder muscles [1-6].

Some studies report no physiological advantage with cambered wheels [7], whereas others have shown a significant decrease in energy costs [7-8]. Past studies had design limitations that may have influenced the varying outcomes. Wheeling efficiency has been measured while subjects wheeled in a straight line; however, the benefits of camber may only be fully realized when users wheel around obstacles and on side slopes [9]. Additionally, wheeling in a straight line does not truly represent day-to-day wheeling, which requires maneuvering around obstacles. Previous camber studies were conducted on nondisabled subjects with little or no wheeling experience, with the results then extrapolated to persons with

Abbreviations: $\mathrm{AB}=$ inexperienced nondisabled (group), $\mathrm{ABE}=$ experienced nondisabled (group), ANOVA = analysis of variance, $\mathrm{DE}=$ experienced with disabilities (group), $\mathrm{L}=$ lumbar, $\mathrm{LSD}=$ least significant difference, $\mathrm{PCI}=$ physiological cost index, RPE = rating of perceived exertion (scale), $\mathrm{T}=$ thoracic, VAS $=$ visual analog scale, $\dot{\mathrm{V}}_{2}=$ oxygen consumption.

*Address all correspondence to Angeliki Perdios, MSc; BC Children's Hospital, A230-4480 Oak Street, Vancouver, British Columbia, Canada V6H3V4. Email: angie.perdios@ubc.ca DOI: 10.1682/JRRD.2006.08.0097 
disabilities [4,6,10-12]. This method was often used to form larger or more convenient sample sizes, and the results may not be directly applicable. The test subjects' wheeling experience, along with the unique physiological muscle recruitment patterns of individuals with disabilities, may be more significant than previously understood [4,13-15].

The mode of wheelchair testing may also affect the outcome. An individual's wheelchair function includes physiological characteristics, such as aerobic capacity, and skills, such as the ability to maneuver a wheelchair around an obstacle [16]. Therefore, subjects with prior wheeling experience must be tested. Although maximal exercise testing is considered the industry standard for assessing aerobic capacity, the role of such testing is limited in people whose performance may be restricted by pain or fatigue [17]. Kinetic and kinematic methodologies are often used to qualify propulsion efficiency via examination of the interaction of such variables as stroke frequency, contact angle, and the forces exerted on the pushrims [18-19]. Wheelchair ergometers, roller systems, and arm crank tests are convenient methods for laboratory testing but may not accurately assess daily wheelchair propulsion. The optimal test of functioning incorporates the individual's usual aids and orthoses at his or her self-selected speed instead of an imposed speed on a treadmill or ergometer [20].

In addition, individuals with lesions below thoracic (T) level 5 exhibit a linear increase in oxygen consumption $\left(\dot{\mathrm{V}}_{2}\right)$ and heart rate related to work rate [21] because of the preservation of the sympathetic innervations to the heart and respiratory muscles [10,22-30]. $\dot{\mathrm{V}} \mathrm{O}_{2}$ is typically expressed relative to the unit time taken to complete the task (milliliters/kilogram/minute). The rate of oxygen consumed per unit distance traveled (milliliters/kilogram/meter) during that specific task is referred to as oxygen cost or energy cost. If cardiopulmonary assessment tools are unavailable, the physiological cost index (PCI) [31-32] can effectively measure energy cost [32]. Sawatzky et al. defined a linear relationship between oxygen cost as measured by pulmonary gas exchange and PCI as measured by heart rate in subjects with spinal lesions at or below T6 [33].

Although rear-wheel camber may affect the maneuverability or overall energy cost of the wheelchair user, its actual impact on the wheelchair user's energy cost is unknown. Subjects with disabilities are unique in their physiology and resultant exercise responses, and to date, the effects of rear-wheel camber on the energy cost of the experienced wheelchair user with disabilities are varied. In this study, we examined the effects of $0^{\circ}, 3^{\circ}$, and $6^{\circ}$ of camber during steady state overground wheeling in experienced wheelchair users with disabilities, nondisabled subjects with wheelchair experience, and nondisabled subjects without wheelchair experience. The three wheel angles were chosen because they are used most frequently among manual wheelchair users during activities of daily living. We hypothesized that $6^{\circ}$ of camber would be the most efficient camber angle in the subjects with prior manual wheeling experience.

\section{METHODS}

\section{Subjects}

All experimental procedures and protocols were approved by the university and hospital clinical research ethics boards. Thirty-four participants were classified as either experienced wheelchair users with disabilities (DE group, $n=13$ ), nondisabled individuals experienced at manual wheeling (ABE group, $n=11$ ), or nondisabled individuals inexperienced at manual wheeling ( $\mathrm{AB}$ group, $n=10$ ). Exclusion criteria included acute infection; overt cardiovascular, pulmonary, metabolic, or orthopedic complications; or mental illness or deficiency that might have interfered with completion of the protocol. Subjects did not eat, drink, or smoke in the 2 hours preceding the test and voided their bladders immediately before testing. The DE subjects were individuals with a lower-limb disability and a spinal lesion at or below T6, including individuals with spina bifida who were at least 1-year postinjury. The ABE subjects included individuals such as therapists who work with wheelchair users and nondisabled wheelchair athletes who have mastered manual wheelchair propulsion and have regularly used a wheelchair for teaching or sport for at least 1 year. The AB subjects had no prior experience with manual wheeling.

\section{Procedures}

All subjects were fitted with a telemetric heart rate monitor (Polar ${ }^{\circledR}$ Electro S710, Kempele, Finland) for continuous data collection and subsequent calculation of the PCI energy cost measure. Each subject's wheelchair was fitted with a camber bar insert that adjusted the wheels to a preset camber angle. A standard 17 in. chair (Invacare ${ }^{\circledR}$ 
A4 IVC Manual Lightweight Wheelchair, Elyria, Ohio) with interchangeable rear-wheel camber was used to test all nondisabled subjects. The subjects with disabilities used their own A4 or A6 model wheelchairs. Standard $53 \mathrm{~cm}$ wheels with pneumatic tires (100 psi; Primo V-Trak, Long Beach, California) were used.

The testing session was divided into two components: a slalom course and a ramp course. All subjects began with a baseline session of a minimum of 5 min rest. Following this baseline measure, all subjects wheeled at a self-selected steady state pace for 5 consecutive min through a $30 \mathrm{~m}$ slalom course. Four chairs were placed at equal intervals in a straight line and subjects wheeled in slalom fashion around each chair. The investigator recorded the total number of laps completed by each subject for each camber angle and the exact pace per lap.

Following the slalom course, subjects indicated their level of comfort on a continuous visual analog scale (VAS) that ranged from extreme discomfort to extreme comfort [34]. The 14-point Rating of Perceived Exertion (RPE) scale was used to determine the subjects' perceptions of physical stress [35]. Subjects were asked to comment on their central RPE and peripheral RPE. The central RPE value was based on the subjects' perceived exertion with regard to dyspnea. The peripheral RPE was based on the subjects' perceived exertion with regard to arm and shoulder muscular fatigue.

Subjects then completed the ramp course, which tested the stability of the varying camber angles on a slope. Subjects wheeled at their own pace on a smooth stable ramp that was approximately $5 \mathrm{~m}$ long and set at an $8^{\circ}$ incline. Subjects wheeled along the ramp at least twice but were allowed a third pass upon request.

The entire protocol, slalom and ramp portions, was repeated for each camber angle $\left(0^{\circ}, 3^{\circ}\right.$, and $\left.6^{\circ}\right)$. The order of the camber angles was randomly assigned and revealed to the subject after completion of the test. Following the testing of all three camber angles, all subjects were asked about personal camber angle preference. Subjects were asked to compare all three camber angles and answer the following user preference questions: (1) During which trial did you feel most supported/stable in your chair while completing the course? (2) During which trial did you find that the ease of maneuverability was better/ improved? (3) During which trial did you find that your hand comfort on the pushrim was better/improved? and (4) Rank each of the three camber angles in terms of preference for comfort and maneuverability. Cardiopulmo- nary measures were continuously collected on 10 of the subjects with disabilities with a portable pulmonary gas exchange system (Cosmed K4b2, Rome, Italy) following standard calibration procedures on ambient air.

\section{Calculations and Statistical Analysis}

We performed all statistical analyses using a commercially available computer program (Statistical Package for the Social Sciences, version 11.0, Chicago, Illinois). Descriptive characteristics for all subjects were generated.

The PCI was derived from the steady state heart rate recorded by the heart rate monitor and from the subjects' average velocity during steady state wheeling. We calculated PCI by first subtracting the average steady state working heart rate from the average resting heart rate and then dividing this value by the subjects' average velocity during slalom wheeling. The resultant value was expressed in beats per meter traveled.

A $3 \times 3$ repeated measures analysis of variance (ANOVA) was conducted for the PCI of the three camber angles $\left(0^{\circ}, 3^{\circ}\right.$, and $\left.6^{\circ}\right)$ and for the three subject groups (DE, $\mathrm{ABE}$, and $\mathrm{AB}$ ). A nonparametric chi-square test and analysis of frequencies were conduced on each subject's user preference responses for $0^{\circ}, 3^{\circ}$, and $6^{\circ}$ of camber. A one-way repeated measures ANOVA was conducted for cardiopulmonary measures. A one-way repeated measures ANOVA was conducted for central RPE and peripheral RPE across all groups for all camber angles. To determine whether comfort level differed during slalom wheeling, we conducted a $3 \times 3$ repeated measures ANOVA among the three camber angles $\left(0^{\circ}, 3^{\circ}\right.$, and $\left.6^{\circ}\right)$ and the three subject groups (DE, $\mathrm{ABE}$, and $\mathrm{AB}$ ). Post hoc analysis was performed with the Fisher's least significant difference (LSD) method. All data are expressed as mean \pm standard deviation.

We conducted a pilot study on five nondisabled individuals with wheeling experience to determine statistical power. The PCI was used as a measure of energy cost during the slalom wheeling course. The values for PCI at $0^{\circ}, 3^{\circ}$, and $6^{\circ}$ of camber were $0.42,0.42$, and 0.32 , respectively. Based on a potential 25 percent reduction in energy cost with $6^{\circ}$ of camber, as compared with $0^{\circ}$ and $3^{\circ}$, a sample size of 10 was calculated for each group, with a statistical power value of 0.8 . 


\section{RESULTS}

Anthropometric data for all subjects are shown in Table 1. The specific lesion levels for the 13 subjects with disabilities were $\mathrm{T} 6=1, \mathrm{~T} 7=1, \mathrm{~T} 8=2, \mathrm{~T} 9=1, \mathrm{~T} 10=1$, $\mathrm{T} 11=2$, T12 = 1, lumbar (L) $1=2, \mathrm{~L} 3=1$, and L4 = 1 . Resting heart rate and $\dot{\mathrm{V}} \mathrm{O}_{2}$ values for 10 subjects with disabilities are listed in Table 2. No significant differences were detected between the three camber angles in the 10 subjects with disabilities assessed for cardiopulmonary measures with the portable metabolic cart (Table 3). No significant differences were detected between the three groups for energy cost, as represented by PCI (Table 4). Central RPE $(p=0.43)$ and peripheral RPE $(p=0.13)$ were not significantly different for any of the camber angles across all groups. A significant difference was detected with the VAS ( $p=0.029$ ); however, Fisher's LSD post hoc analysis showed no significant differences. A difference in camber angle preference based on user preference across all groups tested was found (Table 5). The $6^{\circ}$ camber angle emerged as the angle most preferred in terms of stability on a side slope, maneuverability in slalom, hand comfort on the pushrims, and overall angle preference. Subjects were twice as likely to choose $6^{\circ}$ over $0^{\circ}$ in terms of stability, hand comfort, and overall preference. For ease of maneuverability, subjects were five times as likely to choose $6^{\circ}$ over $0^{\circ}$.

\section{DISCUSSION}

\section{Energy Cost}

We tested individuals with and without disabilities and with varying levels of manual wheeling experience to determine whether camber angle affected wheeling efficiency. No differences in energy expenditure were detected among $0^{\circ}, 3^{\circ}$, and $6^{\circ}$ of rear-wheel camber angle for any of the groups either by the respiratory measures in our DE group or by the PCI in all subjects. Cardiopulmonary measures during steady state wheeling did not detect any difference among the three most commonly used and tested camber angles. Our study results support the research of Veeger et al. [8], who found no significant physiological effect of camber during wheeling on an ergometer where it could not be concluded that rear-wheel camber was physiologically advantageous to vertically placed wheels. In contrast, the results of this study refute

\section{Table 1.}

Anthropometric data (mean \pm standard deviation) for wheelchair users with disabilities and nondisabled individuals with and without manual wheeling experience.

\begin{tabular}{lcccc}
\hline \multicolumn{1}{c}{ Group } & $\boldsymbol{n}$ & $\begin{array}{c}\text { Age } \\
\mathbf{( y r )}\end{array}$ & $\begin{array}{c}\text { Height } \\
\mathbf{( c m )}\end{array}$ & $\begin{array}{c}\text { Weight } \\
\mathbf{( k g )}\end{array}$ \\
\hline With Disabilities & 13 & $32 \pm 9$ & $170 \pm 12$ & $65 \pm 16$ \\
Nondisabled & 11 & $35 \pm 8$ & $178 \pm 9$ & $73 \pm 9$ \\
$\quad$ Experienced & & & & \\
$\begin{array}{l}\text { Nondisabled } \\
\quad \text { Inexperienced }\end{array}$ & 10 & $33 \pm 10$ & $171 \pm 7$ & $64 \pm 10$ \\
\hline \hline
\end{tabular}

Table 2.

Oxygen uptake $\left(\dot{\mathrm{VO}}_{2}\right)(\mathrm{mL} / \mathrm{kg} / \mathrm{min})$ and heart rate $(\mathrm{HR})$ (beats $\left./ \mathrm{min}\right)$ for wheelchair users with disabilities at rest and $0^{\circ}, 3^{\circ}$, and $6^{\circ}$ of rear-wheel camber.

\begin{tabular}{|c|c|c|c|c|c|c|c|c|}
\hline \multirow{2}{*}{ Subject } & \multicolumn{2}{|c|}{ Rest } & \multicolumn{2}{|c|}{$\mathbf{0}^{\circ}$} & \multicolumn{2}{|c|}{$3^{\circ}$} & \multicolumn{2}{|c|}{$6^{\circ}$} \\
\hline & $\dot{\mathrm{V}} \mathrm{O}_{2}$ & HR & $\dot{\mathrm{V}} \mathrm{O}_{2}$ & HR & $\dot{\mathrm{V}} \mathrm{O}_{2}$ & HR & $\dot{\mathrm{V}} \mathrm{O}_{2}$ & HR \\
\hline D1 & 3.1 & 71.4 & 8.4 & 88.1 & 7.5 & 85.2 & 7.1 & 85.4 \\
\hline D3 & 5.2 & 92.2 & 17.3 & 140.8 & 19.7 & 157.9 & 18.2 & 148.8 \\
\hline D4 & 2.5 & 67.0 & 10.9 & 108.1 & 11.5 & 111.5 & 11.8 & 106.2 \\
\hline D7 & 3.7 & 82.9 & 8.1 & 133.3 & 8.6 & 130.2 & 9.1 & 131.5 \\
\hline D8 & 4.4 & 100.5 & 15.2 & 132.8 & 15.2 & 131.8 & 12.1 & 119.5 \\
\hline D9 & 3.7 & 71.0 & 9.7 & 97.9 & 10.3 & 98.8 & 9.9 & 98.3 \\
\hline D10 & 6.5 & 96.6 & 14.5 & 148.8 & 14.9 & 143.2 & 17.1 & 138.6 \\
\hline
\end{tabular}


Table 3.

Mean cardiopulmonary values for wheelchair users with disabilities across $0^{\circ}, 3^{\circ}$, and $6^{\circ}$ of rear-wheel camber.

\begin{tabular}{lcccc}
\hline $\begin{array}{l}\text { Cardiopulmonary } \\
\text { Measure }\end{array}$ & $\mathbf{0}^{\circ}$ & $\mathbf{3}^{\circ}$ & $\mathbf{6}^{\circ}$ & $\boldsymbol{p}$-Value \\
\hline $\begin{array}{l}\text { Breathing Frequency } \\
\text { (breaths/min) }\end{array}$ & 27.4 & 29.2 & 25.8 & 0.14 \\
$\begin{array}{l}\text { Tidal Volume } \\
\quad(\mathrm{mL} / \mathrm{min})\end{array}$ & 0.87 & 0.89 & 0.81 & 0.42 \\
$\begin{array}{l}\text { Ventilation } \\
\quad(\mathrm{mL} / \mathrm{min})\end{array}$ & 22.7 & 25.1 & 19.4 & 0.20 \\
$\begin{array}{l}\text { Oxygen Consumed } \\
\quad\left(\mathrm{mL} / \mathrm{O}_{2} / \text { min) }\right.\end{array}$ & 636.5 & 714.1 & 527.9 & 0.21 \\
$\begin{array}{c}\text { Carbon Dioxide Expired } \\
\text { (mL/CO } 2 \text { /min) }\end{array}$ & 648.0 & 697.3 & 500.9 & 0.24 \\
$\begin{array}{l}\text { Respiratory } \\
\text { Exchange Ratio }\end{array}$ & 1.0 & 0.98 & 0.97 & 0.06 \\
\hline \hline
\end{tabular}

Table 4.

Mean \pm standard deviation physiological cost index (beats $/ \mathrm{m}$ ) across three rear-wheel camber angles $(n=34)$.

\begin{tabular}{lccc}
\hline Group & $\mathbf{0}^{\circ}$ & $\mathbf{3}^{\circ}$ & $\mathbf{6}^{\circ}$ \\
\hline With Disabilities & $0.35 \pm 0.2$ & $0.34 \pm 0.2$ & $0.35 \pm 0.2$ \\
Nondisabled & $0.42 \pm 0.1$ & $0.44 \pm 0.1$ & $0.40 \pm 0.1$ \\
$\quad$ Experienced & & & \\
$\quad$ Nondisabled & $0.33 \pm 0.1$ & $0.36 \pm 0.2$ & $0.32 \pm 0.1$ \\
$\quad$ Inexperienced & & & \\
\hline \hline
\end{tabular}

Table 5.

Frequency of user preference in four categories for three rear-wheel camber angles $(n=27)$.

\begin{tabular}{llll}
\hline User Preference Category & $\mathbf{0}^{\circ}$ & $\mathbf{3}^{\circ}$ & $\mathbf{6}^{\circ}$ \\
\hline Side-Slope Stability & 6 & 8 & 12 \\
Maneuverability in Slalom & 3 & 8 & 15 \\
Pushrim Comfort & 6 & 8 & 12 \\
Overall Preference & 6 & 8 & 13 \\
\hline \hline
\end{tabular}

Buckley and Bhambhani's work that showed increased physiological responses with increasing camber [7].

The two measures of RPE, peripheral and central, were analyzed with camber angle to determine whether subject perception of exertion differed between camber angles. No significant difference was found for peripheral RPE across camber angles for any of the groups tested. A statistically significant difference was found for central RPE across camber angles $(p=0.04)$. However, post hoc analyses showed that no true significant difference existed. The results of this component of the study are consistent with Buckley and Bhambhani's findings that no significant changes were observed in the central and peripheral RPE with camber angle during low-intensity wheelchair exercise [7].

The upper limb was not designed to bear the repetitive strain generated during manual wheelchair propulsion [36]. As a result of the increased demand on these relatively small forearm muscles in people with lowerlimb disabilities, problems such as insufficient muscle perfusion, repetitive strain injuries, and early onset of fatigue become particularly critical [30,37]. Although this study showed no physiological evidence to support increased camber for improved energy cost, camber affected the wheelchair users' perception of comfort, which is an important component of wheeling.

\section{User Preference}

Camber improves maneuverability and stability on a side slope $[1-2,38]$. The results of the user preference questions support this finding. A comparison of the three camber angles revealed that $6^{\circ}$ was most preferred in all four domains questioned. Subjects were twice as likely to choose $6^{\circ}$ over $0^{\circ}$ for increased stability on a side slope, improved hand comfort on the pushrims, and overall preference. In terms of ease of maneuverability around the slalom course obstacles, the subjects were five times as likely to choose $6^{\circ}$ over $0^{\circ}$ of camber. The VAS data did not support the results of the user preference questionnaire. Since the VAS measured overall comfort during wheeling, one would assume that the results would follow the user preference and that $6^{\circ}$ would emerge as the most comfortable camber angle during wheeling. However, no difference in comfort was detected. If the VAS were a discrete rather than a continuous scale, results might have been more consistent with the other user preference findings.

\section{Comparative Analysis}

Our goal was to test three commonly prescribed camber angles and determine the most efficient angle during normal manual wheeling. Efficiency can be quantified a number of ways with biomechanical measures of propulsion intensity, stroke frequency, or stroke angle [39] or physiologic measures. In this study, cardiopulmonary measures validly assessed exertion and, thus, efficiency. From a practical perspective, the importance of the user preference results should not be overlooked. During normal 
wheeling, when user effort is well below maximum, perhaps user preference results more accurately assess efficiency. An individual with disabilities relies on his or her wheelchair as a tool for negotiating the environment, for mobility, and in reality, for freedom. The results of the user preference component provide useful information not only for the individual with disabilities seeking increased comfort and improved maneuverability in his or her environment but also for the healthcare professional making seating recommendations. If a physiological difference in energy cost truly does not exist, then rehabilitation therapists and wheelchair seating specialists can confidently prescribe a camber angle based on the client's personal comfort. That is, they should not be concerned that the wheelchair user will be increasing his or her energy cost when wheeling under steady state conditions with a decreased rear-wheel camber angle.

Although no differences in energy cost emerged, perhaps slight variations in heart rate or $\dot{\mathrm{V}} \mathrm{O}_{2}$ were not detected by the test design or measurement tools. While these slight differences were not detected by traditional physiological equipment, they may have been felt by the subjects and contributed to their selection of $6^{\circ}$ as most comfortable. IJzerman and Nene reported that PCI is considered feasible if the differences in energy requirements that must be detected actually can be detected with sufficient statistical power [40]. A similar problem may have occurred in the present study. The PCI is a feasible tool for measuring energy cost; however, since the PCI is based on heart rate, the minor changes in camber angle may not have been significant enough to elicit a change in heart rate. We are not certain whether a difference in camber angle truly has no effect on energy cost or whether the test simply was not sensitive enough to detect any small differences, especially at the submaximal speed of wheeling [8]. The slalom course was designed to highlight the presence of camber. However, the three different camber angles may not have created an incremental workload sufficient to alter the intensity of wheeling. Although the physiological measures of oxygen cost and PCI do not support $6^{\circ}$ of camber as a more energy efficient wheelchair setup, small differences between camber angles may not be detectable by traditional energy cost measures, especially when the subject is wheeling submaximally. Small undetectable differences may explain why we found no differences in the respiratory measures of our DE subjects tested with the Cosmed K4b2 metabolic cart.

\section{Limitations and Future Recommendations}

The results of this study can only be extrapolated to individuals with lesions at or below T6 who use a wheelchair designed to accommodate camber changes via a camber bar. Another limitation to this study design is that we tested the $A B$ and $A B E$ subjects in a standardized wheelchair and the DE subjects in their own wheelchairs, which invariably affected the results. However, since our intention was to test the subjects in a situation that reflected daily wheeling dynamics, we accepted this potential contamination of the results. Since subjects completed the entire test at a self-selected pace, no incremental work protocol existed, so we can only conclude that camber angle has no effect during steady state wheeling at a self-selected pace. Future studies could use an incremental protocol to quantify changes in energy cost across varying workloads, using $\dot{\mathrm{V}}_{2}$ analysis on all subjects. Combined with a resistor mounted to the rear wheel, this incremental protocol would more comprehensively analyze energy cost over varying speeds and work outputs than reliance on subjects' self-selected steady state pace. Irrespective of the direction of future studies involving camber, we recommend that the wheeling protocol attempt to replicate day-to-day wheeling demands that emphasize the effects of rear-wheel camber angle.

\section{CONCLUSIONS}

Based on the results of this study, rear-wheel camber angle does not affect the energy expenditure during manual wheelchair propulsion. The individual manual wheelchair user's perceived level of comfort should be the determining factor in rear-wheel camber selection.

\section{ACKNOWLEDGMENTS}

This material is the result of work jointly supported with resources from the Natural Sciences and Engineering Research Council and The Hydrocephalus and Spina Bifida Association of British Columbia.

The authors have declared that no competing interests exist. 


\section{REFERENCES}

1. Trudel G, Kirby RL, Bell AC. Mechanical effects of rearwheel camber on wheelchairs. Assist Technol. 1995;7(2): 79-86. [PMID: 10159861]

2. Denison I, Shaw J, Zuyderhoff R. The effect of components on manual wheelchair performance. In: Denison I, Shaw J, Zuyderhoff R, editors. Wheelchair selection. British Columbia rehabilitation equipment evaluation. 1st ed. Vancouver (British Columbia, Canada): BC Rehab; 1994. p. 31-40.

3. Cooper RA. Wheelchair selection and configuration. New York (NY): Demos Medical Publishing; 1998.

4. Van der Woude LH, Veeger HE, Dallmeijer AJ, Janssen TW, Rozendaal LA. Biomechanics and physiology in active manual wheelchair propulsion. Med Eng Phys. 2001;23(10):713-33. [PMID: 11801413]

5. Hilbers PA, White TP. Effects of wheelchair design on metabolic and heart rate responses during propulsion by persons with paraplegia. Phys Ther. 1987;67(9):1355-58.

[PMID: 3628489]

6. Ruggles DL, Cahalan TD, An KN. Biomechanics of wheelchair propulsion by able-bodied subjects. Arch Phys Med Rehabil. 1994;75(5):540-44. [PMID: 8185446]

7. Buckley SM, Bhambhani YN. The effects of wheelchair camber on physiological and perceptual responses in younger and older men. Adapt Phys Activ Q. 1998;15:15-24.

8. Veeger D, Van der Woude LH, Rozendaal RH. The effect of rear wheel camber in manual wheelchair propulsion. J Rehabil Res Dev. 1989;26(2):37-46. [PMID: 2724151]

9. Brubaker CE, McLaurin CA, McClay IS. Effects of side slope on wheelchair performance. J Rehabil Res Dev. 1986; 23(2):55-58. [PMID: 3723426]

10. Glaser RM, Sawka MN, Brune MF, Wilde SW. Physiological responses to maximal effort wheelchair and arm crank ergometry. J Appl Physiol. 1980;48(6):1060-64. [PMID: 7380703]

11. Reid M, Lawrie AT, Hunter J, Warren PM. The effect of steering on the physiological energy cost of wheelchair propulsion. Scand J Rehabil Med. 1990;22(3):139-43. [PMID: 2244191]

12. Rudins A, Laskowski ER, Growney ES, Cahalan TD, An $\mathrm{KN}$. Kinematics of the elbow during wheelchair propulsion: A comparison of two wheelchairs and two stroking techniques. Arch Phys Med Rehabil. 1997;78(11):1204-10. [PMID: 9365350]

13. Harburn KL, Spaulding SJ. Muscle activity in the spinal cord-injured during wheelchair ambulation. Am J Occup Ther. 1986;40(9):629-36. [PMID: 3766686]

14. Keyser RE, Rodgers MM, Gardner ER, Russell PJ. Oxygen uptake during peak graded exercise and single-stage fatigue tests of wheelchair propulsion in manual wheel- chair users and the able-bodied. Arch Phys Med Rehabil. 1999;80(10):1288-92. [PMID: 10527089]

15. Patterson P, Draper S. Selected comparisons between experienced and non-experienced individuals during manual wheelchair propulsion. Biomed Sci Instrum. 1997;33:477-81.

[PMID: 9731406]

16. Webster JS, Cottam G, Gouvier WD, Blanton P, Beissel GF, Wofford J. Wheelchair obstacle course performance in right cerebral vascular accident victims. J Clin Exp Neuropsychol. 1988;11(2):295-310. [PMID: 2925837]

17. Noonan V, Dean E. Submaximal exercise testing: Clinical application and interpretation. Phys Ther. 2000;80(8):782-807. [PMID: 10911416]

18. Consortium for Spinal Cord Medicine. Preservation of upper limb function following spinal cord injury: A clinical practice guideline for health-care professionals. Washington (DC): Paralyzed Veterans of America; 2005.

19. Vanlandewijck Y, Spaepen AJ, Lysens RJ. Wheelchair propulsion efficiency: Movement pattern adaptations to speed changes. Med Sci Sports Exerc. 1994;26(11):1373-81. [PMID: 7837958]

20. Boyd R, Fatone S, Rodda J, Olesch C, Starr R, Cullis E, Gallagher D, Carlin JB, Nattrass GR, Graham K. High- or low-technology measurements of energy expenditure in clinical gait analysis? Dev Med Child Neurol. 1999;41(10): 676-82. [PMID: 10587044]

21. Schmid A, Huonker M, Barturen JM, Stahl F, SchmidtTrucksass A, Konig D, Grathwohl D, Lehmann M, Keul J. Catecholamines, heart rate, and oxygen uptake during exercise in persons with spinal cord injury. J Appl Physiol. 1998;85(2):635-41. [PMID: 9688742]

22. Astrand PO, Rodahl K. Evaluation of physical performance on the basis of tests. In: Textbook of work physiology. New York (NY): McGraw-Hill; 1986. p. 354-87.

23. Bar-On ZH, Nene AV. Relationship between heart rate and oxygen uptake in thoracic level paraplegics. Paraplegia. 1990;28(2):87-95. [PMID: 2235027]

24. Goldsmith R, Miller DS, Mumford P, Stock MJ. The use of long-term measurements of heart rate to assess energy expenditure. J Physiol. 1996;189:35P.

25. Hildebrant G, Voigt E, Bahn D, Berendes B, Kroger J. Energy costs of propelling wheelchair at various speeds: Cardiac response and effect on steering accuracy. Arch Phys Med Rehabil. 1970;51(3):131-36. [PMID: 5440502]

26. Montoye HJ, Taylor HL. Measurement of physical activity in population studies: A review. Hum Biol. 1984;56(2): 195-216. [PMID: 6489978]

27. Rose J, Gamble JG, Medeiros J, Burgos A, Haskell WL. Energy cost of walking in normal children and in those with cerebral palsy: Comparison of heart rate and oxygen uptake. J Pediatr Orthop. 1989;9(3):276-79. [PMID: 2723046] 
JRRD, Volume 44, Number 3, 2007

28. Findley TW, Agre JC. Ambulation in the adolescent with spina bifida. II. Oxygen cost of mobility. Arch Phys Med Rehabil. 1988;69(10):855-61. [PMID: 3052362]

29. Luke A, Maki KC, Barkey N, Cooper R, McGee D. Simultaneous monitoring of heart rate and motion to assess energy expenditure. Med Sci Sports Exerc. 1997;29(1): 144-48. [PMID: 9000168]

30. Kofsky PR, Davis GM, Shephard RJ, Jackson RW, Keene GC. Field testing: Assessment of physical fitness of disabled adults. Eur J Appl Physiol Occup Physiol. 1983; 51(1):109-20. [PMID: 6224676]

31. McGregor J. Rehabilitation ambulatory monitoring. In: Kenedi RM, Paul JP, Hughes J, editors. Disability, proceedings of a seminar on rehabilitation of the disabled. London (England): MacMillan; 1979. p. 159-72.

32. Mattison PG, Hunter J, Spence S. Development of a realistic method to assess wheelchair propulsion by disabled people. Int J Rehabil Res. 1989;12(2):137-45. [PMID: 2625374]

33. Sawatzky BJ, Miller WC, Denison I. Measuring energy expenditure using heart rate to assess the effects of wheelchair tyre pressure. Clin Rehabil. 2005;19(2):182-87. [PMID: 15759533]

34. DiGiovine MM, Cooper R, Boninger ML, Lawrence BM, VanSickle DP, Rentschler AJ. User assessment of manual wheelchair ride comfort and ergonomics. Arch Phys Med Rehabil. 2000;81(4):490-94. [PMID: 10768541]

35. Borg G. Perceived exertion as an indicator of somatic stress. Scand J Rehabil Med. 1970;2(2):92-98. [PMID: 5523831]

36. Sawatzky BJ, Denison I, Kim WO. Rolling, rolling, rolling. Rehabil Manag. 2002;15(6):36-39. [PMID: 12741267]

37. Mukherjee G, Samanta A. Physiological response to the ambulatory performance of hand-rim and arm-crank propulsion systems. J Rehabil Res Dev. 2001;38(4):391-99. [PMID: 1156492]

38. Trudel G, Kirby RL, Ackroyd-Stolarz SA, Kirkland S. Effects of rear-wheel camber on wheelchair stability. Arch Phys Med Rehabil. 1997;78(1):78-81. [PMID: 9014962]

39. Kotajarvi BR, Basford JR, An KN, Morrow DA, Kaufman KR. The effect of visual biofeedback on propulsion effectiveness of experienced wheelchair users. Arch Phys Med Rehabil. 2006;87(4):510-15. [PMID: 16571390]

40. IJzerman MI, Nene AV. Feasibility of the physiological cost index as an outcome measure for the assessment of energy expenditure during walking. Arch Phys Med Rehabil. 2002;83(12):1777-82. [PMID: 12474186]

Submitted for publication September 15, 2006. Accepted in revised form January 23, 2007. 